\title{
Laminar perfusion imaging with zoomed arterial spin labeling at 7 Tesla
}

Xingfeng Shao ${ }^{\mathrm{a}}$, Fanhua Guo ${ }^{\mathrm{b}}$, Qinyang Shou ${ }^{\mathrm{a}}$, Kai Wanga ${ }^{\mathrm{a}}$, Kay Jann ${ }^{\mathrm{a}, \mathrm{c}}$, Lirong Yan ${ }^{\mathrm{a}, \mathrm{c}}$, Arthur W. Toga $^{d}$, Peng Zhang ${ }^{b}$ and Danny JJ Wang ${ }^{a, c,}{ }^{*}$

aLaboratory of FMRI Technology (LOFT), USC Stevens Neuroimaging and Informatics Institute, Keck School of Medicine, University of Southern California, Los Angeles, CA 90033, USA

'State Key Laboratory of Brain and Cognitive Science, Beijing MRI Center for Brain Research, Institute of Biophysics, Chinese Academy of Sciences, Beijing 100101, China

'Department of Neurology, Keck School of Medicine, University of Southern California, Los Angeles, CA 90033, USA

'Laboratory of Neuroimaging, USC Stevens Neuroimaging and Informatics Institute, Keck School of Medicine, University of Southern California, Los Angeles, CA 90033, USA.

${ }^{*}$ Correspondence: Danny JJ Wang

Email: JJ.Wang@loni.usc.edu

Author Contributions: X.S. implemented the MRI pulse sequence, conducted the experiments and data analysis. D.W., P.Z and A.T. provided advice on experimental design and research direction. X.S., D.W. and P.Z. designed the experiments. F.G., Q.S., K.W., L.Y. and K.J. provided input on the design of the analysis. All authors wrote the manuscript.

\section{Keywords:}

Laminar fMRI; Perfusion; Arterial spin labeling; Neural circuit; Visual spatial attention; Ultrahigh field; 


\begin{abstract}
Laminar $\mathrm{AMRI}$ based on BOLD and CBV contrast at ultrahigh magnetic fields has been applied for studying the dynamics of mesoscopic brain networks. However, the quantitative interpretations of BOLD/CBV fMRI results are confounded by different baseline physiology across cortical layers. Here we introduce a novel 3D zoomed pseudo-continuous arterial spin labeling technique at $7 \mathrm{~T}$ that offers the unique capability for quantitative measurements of laminar cerebral blood flow (CBF) both at rest and during task activation with high spatial specificity and sensitivity. We found arterial transit time in superficial layers is $\sim 100 \mathrm{msec}$ shorter than in middle/deep layers revealing the dynamics of labeled blood flowing from pial arteries to downstream microvasculature. Resting state CBF peaked in the middle layers which is highly consistent with microvascular density measured from human cortex specimens. Finger tapping induced a robust two-peak laminar profile of CBF increases in the superficial (somatosensory and premotor input) and deep (spinal output) layers of M1, while finger brushing task induced a weaker CBF increase in superficial layers (somatosensory input). We further demonstrated that top-down attention induced a predominant CBF increase in deep layers and a smaller CBF increase on top of the lower baseline CBF in superficial layers of V1 (feedback cortical input), while bottom-up stimulus driven activity peaked in the middle layers (feedforward thalamic input). These quantitative laminar profiles of perfusion activity suggest an important role of M1 superficial layers for the computation of finger movements, and that visual attention may amplify deep layer output to the subcortex.
\end{abstract}

\title{
Significance Statement
}

CBF or microvascular perfusion measured by arterial spin labeling (ASL) is a key parameter for in vivo assessment of neurovascular function. Compared to BOLD or VASO fMRI, ASL perfusion contrast offers the unique capability for quantitative CBF measurements both at baseline and during task activation, which is critical for quantitative estimation of metabolic activities tightly related to neuronal activation. We proposed a zoomed 3D ASL technique at 7T for laminar perfusion imaging with high spatial specificity and sensitivity. This technique is able to differentiate and quantify the input/output and feedforward/feedback activities of human motor and visual cortex, thereby providing an important tool for quantitative assessment of neurovascular function and metabolic activities of neural circuits across cortical layers. 


\section{Main Text}

\section{Introduction}

It was discovered a century ago that the neocortex in mammals is arranged in six layers that vary across different cortical areas $(1,2)$. This observation formed the basis for the parcellation of human cortex into separate areas, and further assigned feedforward and feedback pathways to different cortical layers $(3,4)$. Blood oxygen level dependent (BOLD) fMRI is the most widely used noninvasive imaging modality for studying the dynamics of macroscopic brain networks and mesoscopic brain circuits. It is now feasible to achieve a sub-millimeter spatial resolution using BOLD fMRI at the level of cortical layers at ultrahigh magnetic fields (UHF) of $7 \mathrm{~T}$ and above (5-7). However, the complex interplay between cerebral blood flow (CBF), cerebral blood volume (CBV) and oxygen metabolism hampers quantitative interpretations of the BOLD signal across cortical layers or depths with different baseline physiology (8). The BOLD signal is also susceptible to contaminations of pial veins on the cortical surface that significantly confounds laminar $f M R I$. Compared to conventional gradient-echo T2*-weighted BOLD, emerging $\mathrm{fMRI}$ methods based on T2-weighted BOLD and CBV contrast have been shown to more precisely map the laminar activities in both animal and human studies (8-13). In particular, vascular space occupancy (VASO) based CBV fMRI at $7 \mathrm{~T}(14,15)$ is able to differentiate specific activations in different layers of human motor cortex (M1), as well as the directional functional connectivity of M1 with somatosensory and premotor areas (9). However, VASO only measures relative CBV changes that may be confounded by different baseline CBV values across cortical layers.

CBF or perfusion measured by arterial spin labeling (ASL) is a key parameter for in vivo assessment of neurovascular function. The ASL signal is localized close to the site of neural activation as most of the labeled arterial water exchanges with tissue water in capillaries (16). Optical imaging in animals provides evidence that CBF can be precisely and rapidly regulated on an extremely fine scale at the level of capillaries (17-20). Compared to BOLD and CBV fMRI, ASL perfusion contrast offers the unique capability for quantitative CBF measurements both at rest and during task activation, which is critical for quantitative estimation of metabolic activities tightly related to neuronal activation. UHF ASL has the dual benefits of increased signal-to-noise ratio (SNR) that scales with $\mathrm{B}_{0}$ field and prolonged tracer half-life (blood $\mathrm{T} 1=\sim 2.1 \mathrm{sec}$ at $\left.7 \mathrm{~T}\right)(21,22)$, and therefore may overcome the major limitation of ASL in terms of low SNR. To date, however, the capability for in vivo mapping of microvascular perfusion at laminar level remains largely nonexistent, mainly due to the low sensitivity and technical challenges of performing high-resolution ASL at ultrahigh fields.

In this study, we introduced a novel zoomed 3D pseudo-continuous ASL ( $\mathrm{pCASL}$ ) technique at 7T with high spatial resolution (1-mm isotropic) and sensitivity to characterize layer-dependent resting and task activation induced perfusion activity in the human motor and visual cortices. For the first time, multi-delay pCASL was applied to measure variations of arterial transit time (ATT) and resting state CBF across cortical layers, illustrating the dynamics of labeled blood flowing from pial arteries, arterioles to downstream microvasculature in the middle layers of cerebral cortex (23). Zoomed pCASL at the optimal post-labeling delay was then applied on the motor cortex for detecting and quantifying the layer-dependent activity of M1 during finger tapping and finger brushing (SI Fig. $\mathrm{S} 1 \mathrm{~A})$. Previous studies have shown that somatosensory and premotor input to M1 largely terminates in the superficial layers (II/III), while cortico-spinal output originates predominantly in the deep layers $(\mathrm{Vb} / \mathrm{VI})(24-26)$. However, the precise quantitative sensory input and motor output of $\mathrm{M} 1$ remain to be determined. The optimal zoomed $\mathrm{pCASL}$ protocol was further applied on the visual cortex to quantify the absolute and relative perfusion changes across cortical layers in response to a visual spatial attention task in V1. While visual attention has been shown to operate through feedback connections along descending visual pathways involving both superficial and deep layers of $\mathrm{V} 1$, a quantitative measure is required to evaluate the relative contribution of deep and superficial 
layers in top-down attention (SI Fig. S1B). In this study, we specifically addressed these unsolved neuroscientific questions using zoomed pCASL perfusion fMRI at 7T.

\section{Results}

\section{Zoomed pCASL protocol at $7 T$}

Applying pCASL at UHF is challenging due to a high specific absorption ratio (SAR) level of RF power, as well as the $\mathrm{B}_{1}{ }^{+}$drop and $\mathrm{B}_{0}$ inhomogeneity that affect labeling efficiency (27). We developed an innovative zoomed 3D pCASL technique to achieve a high labeling efficiency and spatial resolution without exceeding the SAR limit. A segmented 3D inner-volume gradient and spin-echo (GRASE) sequence was applied for zoomed pCASL perfusion imaging of a 3D slab of $100 \times 50 \times 24 \mathrm{~mm}^{3}$ with a high resolution of isotropic $1 \mathrm{~mm}^{3}$. The flip angels (FA) of GRASE refocusing pulses and scheme for segmented acquisition were optimized along with a single background suppression pulse to minimize spatial blurring, physiological noise and SAR (Fig.1A). The workflow was streamlined by performing maximal-intensity-projection (MIP) of the T1w structural MRI to visualize intracranial arteries, followed by placing the pCASL labeling plane above the circle of Willis (CoW) and simultaneously perpendicular to the M3 segment of middle cerebral artery (MCA), P2 segment of posterior cerebral artery (PCA) and A2 segment of anterior cerebral artery (ACA) (Fig.1B). Compared to conventional ASL labeling plane at carotid arteries, this labeling location has three advantages: 1) $B_{1}$ and $B_{0}$ fields are more homogeneous around the center of the brain; 2) Blood flow velocity is slower, and the pCASL labeling scheme can be optimized to reduce SAR while maintaining sufficient labeling efficiency; 3) The distance between the labeling plane and cortex of interest is reduced resulting in shortened ATT and TR $(=2.8 \mathrm{sec})$ for perfusion $\mathrm{fMRI}$ scans. With this optimized zoomed 3D pCASL, a high labeling efficiency of $82.1 \%$ can be achieved with an average SAR of $77.4 \pm 8.9 \%$ of the first level limit $(3.2 \mathrm{~W} / \mathrm{kg}$ on head). Another advantage of the proposed technique is perfusion and T2w BOLD contrasts can be concurrently acquired by pairwise subtraction and summation of label and control images (28).

\section{Laminar profile of Resting state ATT and CBF}

Resting state perfusion scans were performed with the optimized zoomed 3D pCASL at 3 PLDs $(500,1000$ and $1500 \mathrm{msec})$ on the motor and visual cortex, respectively. The ASL imaging volumes were manually positioned to be perpendicular to the 'omega' or 'epsilon' shaped hand 'knob' of M1 $(29,30)$, and parallel to a flat portion of the calcarine fissure (31) for motor and visual cortex, respectively. Each imaging volume was acquired with 6 segments in $16.8 \mathrm{sec}$, and 18 pairs of label and control volumes were acquired in $10 \mathrm{~min}$ for each PLD. The acquired ASL control images exhibited high contrast between gray and white matter, and demonstrated sufficient coverage for the premotor, supplementary motor area (SMA), S1 and M1 areas of the motor cortex (Fig. 1A \& Fig. 2A), as well as for $\mathrm{V} 1$ in the calcarine fissure of the visual cortex (Fig. 1A \& Fig. 3A), respectively (Perfusion maps of twenty axial slices are shown in SI Fig. S2). Dynamic resting state perfusion maps at three PLDs and calculated ATT and CBF maps of motor and visual cortex are shown in Fig. 2B-D and Fig. 3B-D respectively. The perfusion maps at the short PLD of 500 msec showed heterogeneous signals at the GM/CSF boundary, indicating insufficient time for the labeled blood to flow from the labeling plane to the cerebral cortex, and thus the majority of ASL signal still remained in pial arteries or arterioles. The perfusion maps at the longer PLD of 1000 and 1500 msec showed more homogeneous signal distribution with minimal signal remaining in CSF. As a result, the laminar profiles (Fig. 2E and Fig. 3E) of the perfusion signals at the PLD of $500 \mathrm{msec}$ peaked at the GM/CSF boundary and monotonically decreased towards deep layers, while the peak perfusion signal at the PLD of 1000 and $1500 \mathrm{msec}$ shifted towards middle layers. These findings are consistent with the laminar profiles of ATT (Fig. 2E and Fig. 3E) which were shortest in superficial layers $(914.7 \pm 12.1 \mathrm{msec}$ in $\mathrm{M} 1$ and $912.3 \pm 4.4 \mathrm{msec}$ in $\mathrm{V} 1$, averaged from four layers at GM/CSF boundary) and increased towards middle to deep layers (1001.4 $\pm 3.4 \mathrm{msec}$ in M1 and 
994.4 $\pm 0.9 \mathrm{msec}$ in $\mathrm{V} 1$, averaged from four layers at GM/WM boundary). Average resting state CBF (corrected for ATT) across cortical depths were $51.9 \pm 11.3 \mathrm{ml} / 100 \mathrm{~g} / \mathrm{min}$ in $\mathrm{M} 1$ and $60.3 \pm 7.2$ $\mathrm{ml} / 100 \mathrm{~g} / \mathrm{min}$ in $\mathrm{V} 1$ with peak CBF observed in a middle layer $(65.6 \mathrm{ml} / 100 \mathrm{~g} / \mathrm{min}$ in $\mathrm{M} 1$ and 68.5 $\mathrm{ml} / 100 \mathrm{~g} / \mathrm{min}$ in V1, Fig. 2E and Fig. 3E). Peak of resting state CBF is slightly shifted towards middle/deep layers in visual cortex likely due to dense vascular network in granular layer (layer 4) which does not exist in motor cortex. To the best of our knowledge, this is the first time that the dynamics of labeled blood flowing from pial arteries, arterioles to downstream microvasculature is shown in vivo on the cerebral cortex. Our results are highly consistent with microvascular density data measured on specimen of human motor and visual cortex (23) (Fig. 2F \& Fig. 3F).

\section{ASL fMRI for quantifying input and output activity in M1}

Perfusion $\mathrm{fMRI}$ scans were acquired at the PLD of $1000 \mathrm{~ms}$ when the majority of the labeled signal arrives at capillary/tissue space and the dynamic ASL signal reaches its peak (Fig. 2E). Fig. 1C shows the two motor tasks, which consisted of unilateral finger tapping (FT, sequential, frequency of $\sim 2 \mathrm{~Hz}$ ) and finger brushing $(\mathrm{FB}, \sim 2 \mathrm{~Hz})$ of the dominant hand. CBF maps at resting state and during the two motor tasks are shown in Fig. 4B. The two motor tasks elicited different laminardependent activation patterns. Strong and medium CBF increases evoked by the FT and FB tasks can be observed along $\mathrm{M} 1$ respectively, as displayed in two zoomed windows (Fig. 4C for absolute CBF change and Fig. 4D for relative CBF change). Both FT induced absolute ( $\triangle \mathrm{CBF}$ ) and percentage (\%CBF) changes show clearly two peaks in deep $(94.8 \pm 3.4 \mathrm{ml} / 100 \mathrm{~g} / \mathrm{min}$ or $159 \pm 7.8 \%)$ and superficial layers $(57.5 \pm 1.4 \mathrm{ml} / 100 \mathrm{~g} / \mathrm{min}$ or $139 \pm 3.8 \%$ ) respectively (Fig. 4E bottom panel, averaged from 5 layers adjacent to peak), consistent with the hypothesis that FT engages neural activity of both somatosensory and premotor input in the superficial layers and motor output in the deep layers (24-26) (SI Fig. S1A). Absolute CBF increase was much larger in the superficial layers than in the deep layers, suggesting that the superficial layers of M1 might involve extensive neural computations integrating the somatosensory and motor planning signals to generate motor output in the deep layers. Interestingly, this finding was not observed in CBV-weighted VASO fMRI of human M1 which showed comparable activation in the superficial and deep layers during FT (24). Statistical significance of the two-peak pattern was validated by $R^{2}$ diff which measures the likelihood that a profile consists of two isolated distributions instead of one. This double peak pattern is highly reliable ( $\mathrm{P}=0.011$ for $\triangle \mathrm{CBF}$ and $\mathrm{P}<0.0002$ for \%CBF, SI Fig. S8) and can be observed in all participants (see individual results in SI appendix, Fig. S3). FB-induced CBF increase was much smaller averaged cross cortical layers compared to that of the FT task. The perfusion response mainly peaked in superficial layers (Fig. 4E bottom panel, $38.8 \pm 2.7 \mathrm{ml} / 100 \mathrm{~g} / \mathrm{min}$ or $69.3 \pm 5.7 \%$ and $15.3 \pm 0.9 \mathrm{ml} / 100 \mathrm{~g} / \mathrm{min}$ or $45.2 \pm 1.5 \%$ in the superficial and deep layers (averaged from 5 layers adjacent to peak) respectively), consistent with the hypothesis that FB primarily engages somatosensory input and minimal motor output (SI Fig. S1A). These results demonstrate the high spatial specificity of ASL, capable of resolving and further quantifying layer-dependent input and output activity in human M1. Both FT and FB induced BOLD signal changes are mostly in superficial layers (Fig. 4E top right). FT induced BOLD response shows a weak increase in deep layers; however, the two-peak pattern was not significant $(P=0.47)$ due to the large BOLD signal change in superficial layers. Furthermore, correction of BOLD signal which was on the order of $1-2 \%$ had minimal effect on perfusion signal changes (Fig. 4E lower right dashed trace). Our results are highly consistent with CBV fMRI data, nevertheless ASL fMRI provides additional measurements of both absolute and relative CBF changes with $\mathrm{FT}$ and $\mathrm{FB}$ inducing $73.6 \pm 12.5 \mathrm{ml} / 100 \mathrm{~g} / \mathrm{min}$ and $26.0 \pm 6.4$ $\mathrm{ml} / 100 \mathrm{~g} / \mathrm{min}$ or $160.6 \pm 28.6 \%$ and $51.5 \pm 22.0 \%$ CBF increase across all cortical depths. This is the first time that such detailed quantitative information on both absolute and relative perfusion activation related to the input and output activity of the motor cortex is visualized in vivo.

\section{ASL fMRI for quantifying feedforward and feedback activity in V1}

We further applied 3D zoomed pCASL for perfusion $\mathrm{fMRI}$ with a visual spatial attention task to resolve and quantify the feedforward and feedback activities of human V1. As shown in Fig. 1D of 
the task paradigm, subjects were required to pay attention to the cued checkerboard pattern presented on either left or right visual field to detect occasional spatial frequency change of the attended stimulus (32). Resting state CBF and visual attention activated CBF maps are shown in Fig. 5B. The attended and unattended visual stimuli induced strong CBF increases localized along the gyri and sulci of visual cortex. Absolute CBF increases to unattended visual stimuli over baseline and attended over unattended stimuli are displayed in two zoomed windows (Fig. 5C). Fig. 5D shows the relative CBF change evoked by unattended visual stimuli over baseline (left column) and the multiplicative effect of attention modulation map (right column) defined as the ratio between CBF increase evoked by top-down attention (attended - unattended) and stimulus-driven response (unattended - baseline). Fig. 6E shows the laminar profile of CBF at rest and with unattended and attended visual stimuli (top left), BOLD changes (top right), absolute and relative CBF changes induced by unattended visual stimuli (middle panel, BOLD corrected relative CBF changes are also shown), absolute CBF changes induced by top-down attention (bottom left), and $\mathrm{CBF}$ and BOLD response to attention modulation (bottom right). Both absolute and relative CBF increase evoked by unattended visual stimuli peak in middle layers, consistent with the hypothesis that that feedforward visual input from the lateral geniculate of the thalamus mainly reaches the middle layers of V1 $(33,34)$ (SI Fig. S1B). Absolute CBF increase evoked by top-down attention shows a large peak in deep layers while the CBF profile of multiplicative attention modulation shows a two-peak pattern ( $P=0.039$ of two-peak pattern significance test, SI Fig. S8) in both deep $(19.0 \pm 1.7 \%$, two-layer average) and superficial layers (14.5 $\pm 1.8 \%$, two-layer average) versus middle layers $(8.3 \pm 0.5 \%$, two-layer average). These results are consistent with the hypothesis that attention operates through feedback pathways involving both deep and superficial layers $(32,33)$ (SI Fig. S1B). The single-peak pattern of unattended visual stimulation and double peak pattern of attention modulation is highly reliable and was observed in all participants (see individual results in SI appendix, Fig. S4). In contrast, BOLD profile of attention modulation is relatively flat across layers with a slight increase towards $G M / C S F$ boundary $(P=0.063$ of two-peak pattern significance test).

\section{Discussion}

We demonstrated that multi-delay ASL can reveal perfusion dynamics and quantify ATT and resting state CBF across cortical layers. Vascular architecture in human brain is specialized to maintain and regulate blood supply through surface pial arterioles (35), the subsurface microvascular bed and penetrating arterioles bridging the surface and subsurface networks (36). We found baseline ATT was shortest at GM and CSF boundaries indicating fast arrival of blood at pial arteries on cortical surfaces. ATT in middle and deep layers were rather homogeneous indicating a fast transit through penetrating arterioles to the subsurface microvascular bed $(23,36,37)$. The laminar profile of baseline CBF exhibited a single peak around the middle layers and monotonically decreased towards deep and superficial layers in both M1 and V1. The CBF profile matches well with the highest capillary density observed in the middle layers as reported in anatomic studies in specimens of human brain tissue $(23,38)$. Additionally, we observed the peak of resting state CBF in visual cortex is more into deep layers as compared to motor cortex, which likely corresponds to a granular layer with high vascular density in visual cortex. Pathological ATT or CBF heterogeneity across cortical layers might be associated with disturbances in endoneurial metabolism or capillary morphology and implicate a number of diseases including stroke (37, 39), ischemia (40), Alzheimer's disease (41), traumatic brain injury (42), and diabetic nephropathy (43). Studying laminar dependent perfusion dynamics may provide meaningful insights into the progression of neurological diseases.

We further demonstrated the sensitivity and spatial specificity of the proposed ASL $F M R I$ in differentiating and quantifying input and output-driven neural activities in M1. Compared to the concurrently acquired T2w BOLD contrast, ASL fMRI was able to detect two separate peaks in superficial and deep layers of M1 (Fig. 4) across all participants (SI Fig. S3), while the two-peak 
pattern was not significant in BOLD signals. This result is highly consistent with VASO FMRI data (14), supporting that both CBF and CBV based laminar $\mathrm{AMRI}$ is able to differentiate input and output activities of human M1. Nevertheless, CBF-based fMRI provides both absolute and relative perfusion activation, thereby more quantitative information on the magnitude of neural activation across cortical layers. In contrast, VASO only measures relative CBV changes that may be confounded by different baseline CBV values across cortical layers. Furthermore, the proposed ASL $f M R I$ is robust to potential BOLD contamination which is on the order of $1-2 \%$. The quantitative measurement of CBF increases to the finger tapping task revealed a larger peak in the superficial layers than in the deep layers, suggesting an important role of M1 superficial layers integrating the input signals from somatosensory and premotor cortex to generate motor output in the deep layers.

ASL fMRI was also applied with a visual spatial attention task to detect and quantify feedforward and feedback activities in the visual cortex. We observed multiplicative attention modulation in both deep and superficial layers while unattended visual stimuli induced peak CBF increase mainly in the middle layers (Fig. 5). Furthermore, the quantitative perfusion changes induced by top-down attention shows a single large peak in the deep layers. The peak of attention modulation in the deep layers was not clearly visible in concurrently acquired T2w BOLD contrast. Our result is highly consistent with the known neuroanatomy of feedforward and feedback visual pathways (27), further supporting the high sensitivity and spatial specificity of CBF-based fMRI. The superficial and deep layers of $\mathrm{V} 1$ receive feedback inputs from higher cortical areas. The superficial layers also send output to higher order visual cortex, while the deep layers send output to the subcortical visual areas including the LGN, pulvinar and SC, etc $(34,44)$. The multiplicative effect of attention modulation in both superficial and deep layers suggests that the control of attention operates thorough descending feedback pathway from higher cortical areas, while the predominant increase of absolute CBF in the deep layers suggest that the effect of attention mainly amplifies output signals to the subcortex (44) (SI Fig. S1B).

Recent studies demonstrated the feasibility of performing ASL fMRI with high spatial resolutions $(45,46)$. At ultra-high fields, ASL has the dual benefits of long tracer half-life determined by blood T1 (47) and a super-linear relationship between SNR and field strength (48), resulting in a $\sim 3$ fold SNR increase at 7T as compared to 3T. However, shorter arterial blood T2 (68 msec) at 7T (49) places a strict limit on the choice of TE to minimize signal loss as well as blurring along slice direction. We achieved minimal TE and echo-train length using zoomed imaging with half FOV along phase encoding direction, partial Fourier acquisition and segmented readout. Physiological noises in dynamic perfusion signals were minimized by a principle component analysis (PCA)based denoising algorithm (50) and signal blurring was further minimized by POCS reconstruction and a SVD based deblurring algorithm. A diagram of the deblurring process is shown in SI Fig. S5. The resultant point-spread function (PSF) of our method was 1.37 pixel which is sharper than or comparable to the PSFs of BOLD and CBV fMRI (51).

Both imaging coverage and temporal resolution of the proposed technique can be further improved by utilizing accelerated image acquisition and constrained reconstructions $(52,53)$. It is also feasible to perform perfusion based functional connectivity analysis of brain network dynamics with increased coverage and temporal resolution (54). In this study we observed strong perfusion based functional connectivity between M1 and associated brain regions including premotor, SMA and S1 (see individual functional connectivity map in SI Fig. S6). The proposed technique can be further extended to obtain concurrent CBF, BOLD and CBV measurements for mapping metabolic activities (55). With the advent of even higher field strength of 10.5T MRI machine (56) and high density array coils (57), the sensitivity and resolution of zoomed pCASL can be further improved.

In conclusion, we demonstrated high spatial specificity and sensitivity of laminar perfusion fMRI using 3D zoomed pCASL at 7T in detecting and quantifying the input versus output and feedforward versus feedback activities of neural circuits in the motor and visual cortices. With the unique capability for quantitative CBF measurements both at baseline and during task activation, high- 
resolution ASL perfusion $\mathrm{fMRI}$ at $7 \mathrm{~T}$ provides an important tool for in vivo assessment of neurovascular function and metabolic activities of neural circuits across cortical layers.

\section{Materials and Methods}

\section{Human participants}

Six right-handed participants (age $=28.2 \pm 3.5$ years, 2 males and 4 females) underwent motor task experiments and six participants with normal or corrected to normal vision (age $=27.7 \pm 3.7$ years, 3 males and 3 females) underwent spatial visual attention experiments, respectively. All subjects refrained from caffeine intake three hours before the scan. All participants provided written informed consents according to a protocol approved by the Institutional Review Board (IRB) of the University of Southern California.

\section{Experimental protocol and session setup}

Experiments were conducted on a 7 Tesla Terra scanner (Siemens Healthineers, Erlangen, Germany), using a single-channel transmit and 32-channel receive head coil (Nova Medical, Wilmington, MA, USA). Third order shimming was performed before ASL scans to improve $B_{0}$ field homogeneity. Head motion was minimized by placing cushions on top and two sides of head and taping participant's chin to coil. Experienced participants were recruited in this study and overall framewise displacement of ASL scans was less than $0.2 \mathrm{~mm}$ (SI Fig. S7).

Imaging parameters for ASL scans were: FOV=100 $\times 50 \mathrm{~mm}^{2}$, 24 slices $(33 \%$ oversampling, 6/8 partial Fourier), 1-mm isotropic resolution, 2 and 3 segments along phase and partition directions, $\mathrm{TE}=26.78 \mathrm{msec}$, echo train length=214.2 $\mathrm{msec}$, labeling duration=1280 $\mathrm{msec}, \mathrm{TR}=2800 \mathrm{msec}$. FAs of 8 refocusing pulses were $120^{\circ}$ and the first FA was increased to $150^{\circ}$ for signal stabilization. Zoomed imaging was achieved by switching excitation and refocusing gradients between slice and phase directions for inner-volume acquisition (Fig.1A). One non-selective HS pulses was used for suppressing background signal. M1 and V1 were identified from uniform (UNI) MP2RAGE $(0.7-\mathrm{mm}$ isotropic resolution, inversion time $=1000 / 3200 \mathrm{~ms}$, scan time $=9 \mathrm{~min} 46 \mathrm{sec}$ ) images. ASL imaging volumes were placed perpendicular to the 'omega' or 'epsilon' shaped hand 'knob' of M1 $(29,30)$ and parallel to a flat portion calcarine fissure (31) for motor and visual task experiments, respectively.

Intracranial vasculature was revealed by maximal-intensity-projection (MIP) of the UNI MP2RAGE images, and pCASL labeling plane was placed above the circle of Willis (CoW) and simultaneously perpendicular to the M3 segment of MCA, P2 segment of PCA and A2 segment of ACA (Fig.1B). Flow velocity at the labeled vessels was measured by ECG-gated phase-contrast MRI in two subjects. Average blood flow velocity at labeled arteries was $18.4 \pm 4.0 \mathrm{~cm} / \mathrm{sec}$. Average B1 at labeling was $74.0 \pm 11.5 \%$ of ideal B1.

Motor and visual task experiments were conducted in two separate sessions. Each session included MP2RAGE, resting state ASL scans at two PLDs (500/1500 msec, $8 \mathrm{~min} 24 \mathrm{sec}$ ) and four runs of motor or visual task ASL scans (10 min $4 \mathrm{sec}$ ) at PLD=1000 msec. Total scan time was less than 70 mins per session. Each task run consisted of nine interleaved blocks $(67.2 \mathrm{sec}$ per block) with counterbalanced order of tasks across participants.

Fig. $1 \mathrm{C}$ shows the paradigm of motor tasks, which consisted of unilateral finger tapping (sequential, frequency of $2 \mathrm{~Hz}$ ) and finger brushing of the dominant hand. Participants were instructed to remain relaxed with their hands facing up during the brushing task. Participants' fingers were brushed back and forth by a custom-made MRI safe brush at a frequency of $2 \mathrm{~Hz}$. Fig. 1D shows the stimuli and paradigm of the visual task. Visual stimuli were generated in MATLAB (Mathworks Inc.) with 
psychophysics toolbox (https://www.psychtoolbox.net/). Stimuli were presented with the BOLDscreen 32 LCD (Cambridge Research Systems Ltd, Rochester, UK) installed at the end of MRI bore. Participants viewed the stimuli through a mirror mounted on top of the head coil. Subjects were required to keep fixation during the experiment. Before stimulus presentation, a central cue was presented at fixation for 1 second, then a pair of counter-phase flickering $(7.5 \mathrm{~Hz}, 50 \%$ contrast) square wave checkerboards patterns (2 cycles per degree) were presented to the left and the right side of fixation. The size of the checkerboard discs were 7 degrees in diameter, presented at an eccentricity of 6 degrees. During the stimulus presentation, the spatial frequency of the two checkerboard patterns changed 7 times randomly and independently. Subjects were asked to pay attention to the cued checkerboard to detect occasional spatial frequency change of the attended stimulus in left or right vision field in two task runs. Successful detection of the cued pattern within 2 secs was recorded by pressing a button box. Average accuracy was $73.7 \pm 13.9 \%$ across participants.

\section{Signal processing}

Partial Fourier reconstruction was done by the Projection Onto Convex Sets (POCS) method (58). Dynamic ASL image volumes from resting state and task ASL runs were realigned and coregistered using SPM12 (Functional Imaging Laboratory, University College London, UK). Spatial weightings were applied for $\mathrm{M} 1$ and $\mathrm{V} 1$ to avoid distortion or imperfect slice profile in FOV boundaries. Average framewise displacement (FD) of resting state and functional ASL runs were $0.08 \pm 0.03$ and $0.19 \pm 0.07 \mathrm{~mm}$ in motor cortex and $0.09 \pm 0.03$ and $0.13 \pm 0.03$ in visual cortex (Parameters of rigid head motion are shown in SI Fig. S7). A boundary based algorithm was used to co-register ASL image volumes and structural MP2RAGE image (59). Control and label images were pairwise subtracted and summed to obtain perfusion and T2w BOLD images respectively (28). Dynamic signal fluctuations induced by motion and physiological noises were minimized by a PCA-based algorithm (50). Signal decay in k-space was prominent in partition direction due to long echo train length. k-space profile along partition direction was estimated by the extended phase graph (EPG) method (60) with inputs of TE, flip angle train and T2 of arterial blood (68 msec (49)). The point spread function (PSF) of perfusion signal blurring along slice direction was calculated by 1-D Fourier transform of the k-space signal. A singular value decomposition (SVD) based deblurring algorithm was implemented to reduce FWHM of PSF from $2.15 \mathrm{~mm}$ to $1.37 \mathrm{~mm}$ (SI Fig. S5).

Resting state $(\mathrm{PLD}=1000 \mathrm{msec})$ and task activation perfusion signals were extracted and recombined from four task ASL runs. ATT and resting state CBF map were simultaneously estimated from resting state perfusion signal at three PLDs using a weighted-delay approach (61). Task activation CBF was calculated according to (62) incorporating ATT. CBF maps with visual attention stimuli in left or right visual fields were calculated, and corresponding hemispheres with and without attention were separated and re-combined to produce attended and unattended CBF maps.

\section{Segmentation of cortical layers in M1 and V1}

ASL images were upscaled to a finer grid of $0.25 \times 0.25 \times 0.5 \mathrm{~mm}^{3}$ resolution to avoid singularities at the edges in angular voxel space. The borderlines of CSF/GM and GM/WM in M1 hand-knob area were manually drawn on ASL control images. Twenty cortical layers of M1 were segmented by LAYNII (63) using the equi-volume layering approach (64). Segmentation of cortical layers in V1 was done on reconstructed surface. MP2RAGE volume was skull removed and segmented into WM, GM and CSF using FreeSurfer (7.1.1). AFNI/SUMA and custom python/matlab codes were used to generate the equi-volume surfaces (https://github.com/herrlich10/mripy) between WM and pial surfaces. Fifteen cortical layers in V1 were then projected back to volume space for analysis. The equi-volume layering approach used in this study has been shown to have higher accuracy than the equi-distance model by taking into account the local curvature of pial and white matter surfaces into account (64). Considering the difference between cortical thickness in M1 ( 4mm) 
and $\mathrm{V} 1$ ( 2-3 $\mathrm{mm}$ ) (65), fifteen and twenty layers were segmented in V1 and M1 respectively. With a nominal $1 \mathrm{~mm}$ resolution, the effective resolution allows it to detect only $\sim 4$ independent data points. Hence, the defined cortical depths do not represent the MRI effective resolution.

\section{Significance test for two-peak activation pattern}

Two-peak patterns were observed in CBF FT activation and attention modulation profiles. To test the significance of the two-peak pattern, we calculated $R^{2}$ diff for each profile. $R^{2}$ diff was defined as the difference between the adjust $R^{2}$ estimated from curve fitting assuming two or single Gaussian distributions. Larger $\mathrm{R}^{2}$ diff indicates the profile can be better described as two peaks instead of a single peak. Statistical significance of the $\mathrm{R}^{2}$ diff was estimated by 5000 Boot strapping runs with random noise in each cortical layer estimated by inter-subject variation. $P$ value was calculated as the probability that $R^{2}$ diff Score of profile can be explained by noise only (SI Fig. S8). $P<0.05$ was considered as significant. Although other measures including Hartigan's dip statistic (HDS), bimodality coefficient (BC), Akaike's information (66) and Larkin's-F-scores $(24,67)$ were proposed for multimodality versus unimodality test, the proposed $\mathrm{R}^{2}$ diff score was well-defined for doublepeak detection and more robust to misclassification.

\section{Acknowledgments}

This work was supported by the National Institute of Health (NIH) grant UH3-NS100614, S10OD025312, R01-NS114382 and R01-EB028297.

\section{References}

1. K. Amunts, K. Zilles, Architectonic Mapping of the Human Brain beyond Brodmann. Neuron 88, 1086-1107 (2015).

2. K. Broadman, Vergleichende Lokalisationslehre der Grosshirnrinde in ihren Prinzipien dargestellt auf Grund des Zellenbaues : Brodmann, K : Free Download, Borrow, and Streaming : Internet Archive (Leipzig, Barth, 1909).

3. S. Shipp, Structure and function of the cerebral cortex. Curr Biol 17, R443-449 (2007).

4. D. J. Felleman, D. C. Van Essen, Distributed hierarchical processing in the primate cerebral cortex. Cereb Cortex 1, 1-47 (1991).

5. P. J. Koopmans, M. Barth, S. Orzada, D. G. Norris, Multi-echo fMRI of the cortical laminae in humans at 7 T. Neuroimage 56, 1276-1285 (2011).

6. J. R. Polimeni, B. Fischl, D. N. Greve, L. L. Wald, Laminar analysis of 7T BOLD using an imposed spatial activation pattern in human V1. Neuroimage 52, 13341346 (2010).

7. K. Cheng, R. A. Waggoner, K. Tanaka, Human ocular dominance columns as revealed by high-field functional magnetic resonance imaging. Neuron 32, 359374 (2001).

8. L. Huber, K. Uludag, H. E. Moller, Non-BOLD contrast for laminar $\mathrm{fMRI}$ in humans: CBF, CBV, and CMRO2. Neuroimage 197, 742-760 (2019).

9. L. Huber et al., High-Resolution CBV-fMRI Allows Mapping of Laminar Activity and Connectivity of Cortical Input and Output in Human M1. Neuron 96, 12531263.e1257 (2017).

10. C. A. Olman et al., Layer-specific fMRI reflects different neuronal computations at different depths in human V1. PLoS One 7, e32536 (2012). 
11. Z. Chen et al., Multi-phase passband balanced SSFP fMRI with $50 \mathrm{~ms}$ sampling rate at 7 Tesla enables high precision in resolving 100 ms neuronal events. Magnetic Resonance Imaging 35, 20-28 (2017).

12. T. Q. Duong, D. S. Kim, K. Ugurbil, S. G. Kim, Localized cerebral blood flow response at submillimeter columnar resolution. Proc Natl Acad Sci U S A 98, 10904-10909 (2001).

13. V. G. Kemper et al., Sub-millimeter T2 weighted fMRI at 7 T: comparison of 3DGRASE and 2D SE-EPI. Front Neurosci 9, 163 (2015).

14. L. Huber et al., Cortical lamina-dependent blood volume changes in human brain at 7 T. Neuroimage 107, 23-33 (2015).

15. H. Lu, X. Golay, J. J. Pekar, P. C. Van Zijl, Functional magnetic resonance imaging based on changes in vascular space occupancy. Magn Reson Med 50, 263-274 (2003).

16. J. A. Detre, J. J. Wang, Technical aspects and utility of $\mathrm{fMRI}$ using BOLD and ASL. Clinical Neurophysiology 113, 621-634 (2002).

17. E. Chaigneau, M. Oheim, E. Audinat, S. Charpak, Two-photon imaging of capillary blood flow in olfactory bulb glomeruli. Proceedings of the National Academy of Sciences 100, 13081-13086 (2003).

18. C. N. Hall et al., Capillary pericytes regulate cerebral blood flow in health and disease. Nature 508, 55-60 (2014).

19. N. B. Hamilton, D. Attwell, C. N. Hall, Pericyte-mediated regulation of capillary diameter: a component of neurovascular coupling in health and disease. Frontiers in neuroenergetics 2, 5 (2010).

20. C. M. Peppiatt, C. Howarth, P. Mobbs, D. Attwell, Bidirectional control of CNS capillary diameter by pericytes. Nature 443, 700-704 (2006).

21. W. Li et al., Quantitative theory for the longitudinal relaxation time of blood water. Magn Reson Med 76, 270-281 (2016).

22. J. Wang et al., Comparison of quantitative perfusion imaging using arterial spin labeling at 1.5 and 4.0 Tesla. Magn Reson Med 48, 242-254 (2002).

23. H. M. Duvernoy, S. Delon, J. Vannson, Cortical blood vessels of the human brain. Brain research bulletin 7, 519-579 (1981).

24. L. Huber et al., High-resolution CBV-fMRI allows mapping of laminar activity and connectivity of cortical input and output in human M1. Neuron 96, 1253-1263. e1257 (2017).

25. T. Mao et al., Long-range neuronal circuits underlying the interaction between sensory and motor cortex. Neuron 72, 111-123 (2011).

26. N. Weiler, L. Wood, J. Yu, S. A. Solla, G. M. Shepherd, Top-down laminar organization of the excitatory network in motor cortex. Nature neuroscience 11, 360-366 (2008).

27. W. C. Wu, M. Fernández-Seara, J. A. Detre, F. W. Wehrli, J. Wang, A theoretical and experimental investigation of the tagging efficiency of pseudocontinuous arterial spin labeling. Magn Reson Med 58, 1020-1027 (2007).

28. E. C. Wong, R. B. Buxton, L. R. Frank, Implementation of quantitative perfusion imaging techniques for functional brain mapping using pulsed arterial spin labeling. NMR in Biomedicine: An International Journal Devoted to the Development and Application of Magnetic Resonance In Vivo 10, 237-249 (1997). 
29. M. Caulo et al., New morphologic variants of the hand motor cortex as seen with MR imaging in a large study population. American Journal of Neuroradiology 28, 1480-1485 (2007).

30. T. Yousry et al., Localization of the motor hand area to a knob on the precentral gyrus. A new landmark. Brain: a journal of neurology 120, 141-157 (1997).

31. P. Daniel, D. Whitteridge, The representation of the visual field on the cerebral cortex in monkeys. The Journal of physiology 159, 203-221 (1961).

32. C. Liu et al., Layer-dependent multiplicative effects of spatial attention on contrast responses in human early visual cortex. Prog Neurobiol, 101897 (2020).

33. D. J. Felleman, D. C. Van Essen, Distributed hierarchical processing in the primate cerebral cortex. Cerebral cortex (New York, NY: 1991) 1, 1-47 (1991).

34. C. Liu et al., Layer-dependent multiplicative effects of spatial attention on contrast responses in human early visual cortex. Progress in Neurobiology, 101897 (2020).

35. C. B. Schaffer et al., Two-photon imaging of cortical surface microvessels reveals a robust redistribution in blood flow after vascular occlusion. PLoS Bio/4, e22 (2006).

36. U. Baran, Y. Li, R. K. Wang, Vasodynamics of pial and penetrating arterioles in relation to arteriolo-arteriolar anastomosis after focal stroke. Neurophotonics 2 , 025006 (2015).

37. N. Nishimura, C. B. Schaffer, B. Friedman, P. D. Lyden, D. Kleinfeld, Penetrating arterioles are a bottleneck in the perfusion of neocortex. Proceedings of the National Academy of Sciences 104, 365-370 (2007).

38. F. Lauwers, F. Cassot, V. Lauwers-Cances, P. Puwanarajah, H. Duvernoy, Morphometry of the human cerebral cortex microcirculation: general characteristics and space-related profiles. Neuroimage 39, 936-948 (2008).

39. L. Østergaard et al., The role of the cerebral capillaries in acute ischemic stroke: the extended penumbra model. Journal of Cerebral Blood Flow \& Metabolism 33, 635-648 (2013).

40. L. Østergaard et al., The role of the microcirculation in delayed cerebral ischemia and chronic degenerative changes after subarachnoid hemorrhage. Journal of Cerebral Blood Flow \& Metabolism 33, 1825-1837 (2013).

41. L. Østergaard et al., The capillary dysfunction hypothesis of Alzheimer's disease. Neurobiology of aging 34, 1018-1031 (2013).

42. L. Østergaard et al., Capillary transit time heterogeneity and flow-metabolism coupling after traumatic brain injury. Journal of Cerebral Blood Flow \& Metabolism 34, 1585-1598 (2014).

43. L. Østergaard et al., The effects of capillary dysfunction on oxygen and glucose extraction in diabetic neuropathy. Diabetologia 58, 666-677 (2015).

44. P. Balaram, N. A. Young, J. H. Kaas, Histological features of layers and sublayers in cortical visual areas V1 and V2 of chimpanzees, macaque monkeys, and humans. Eye and brain 6, 5 (2014).

45. S. Kashyap et al., Sub-millimetre resolution laminar fMRI using Arterial Spin Labelling in humans at 7T. bioRxiv (2020).

46. M. Taso, F. Munsch, L. Zhao, D. C. Alsop, Regional and depth-dependence of cortical blood-flow assessed with high-resolution Arterial Spin Labeling (ASL). Journal of Cerebral Blood Flow \& Metabolism, 0271678X20982382.

47. Z. Zuo et al., Turbo-FLASH based arterial spin labeled perfusion MRI at $7 \mathrm{~T}$. PloS one 8 (2013). 
48. R. Pohmann, O. Speck, K. Scheffler, Signal-to-noise ratio and MR tissue parameters in human brain imaging at 3, 7 , and 9.4 tesla using current receive coil arrays. Magnetic resonance in medicine 75, 801-809 (2016).

49. L. C. Krishnamurthy et al., Dependence of blood T2 on oxygenation at 7 T: in vitro calibration and in vivo application. Magnetic resonance in medicine 71, 2035-2042 (2014).

50. H. Zhu, J. Zhang, Z. Wang, Arterial spin labeling perfusion MRI signal denoising using robust principal component analysis. Journal of neuroscience methods 295, 10-19 (2018).

51. A. J. Beckett et al., Comparison of BOLD and CBV using 3D EPI and 3D GRASE for cortical layer functional MRI at $7 \mathrm{~T}$. Magnetic resonance in medicine 84, 31283145 (2020).

52. S. Park, S. Torrisi, J. D. Townsend, A. Beckett, D. A. Feinberg, Highly accelerated submillimeter resolution 3D GRASE with controlled blurring inweighted functional MRI at 7 Tesla: A feasibility study. Magnetic Resonance in Medicine 85, 2490-2506 (2021).

53. S. M. Spann et al., Robust single-shot acquisition of high resolution whole brain ASL images by combining time-dependent 2D CAPIRINHA sampling with spatiotemporal TGV reconstruction. Neurolmage 206, 116337 (2020).

54. K. Jann et al., Functional connectivity in BOLD and CBF data: similarity and reliability of resting brain networks. Neuroimage 106, 111-122 (2015).

55. Y. Yang, H. Gu, E. A. Stein, Simultaneous MRI acquisition of blood volume, blood flow, and blood oxygenation information during brain activation. Magnetic Resonance in Medicine: An Official Journal of the International Society for Magnetic Resonance in Medicine 52, 1407-1417 (2004).

56. M. A. Ertürk et al., Toward imaging the body at 10.5 tesla. Magnetic resonance in medicine 77, 434-443 (2017).

57. N. Petridou et al., Pushing the limits of high-resolution functional MRI using a simple high-density multi-element coil design. NMR in biomedicine 26, 65-73 (2013).

58. E. M. Haacke, E. Lindskogj, W. Lin, A fast, iterative, partial-Fourier technique capable of local phase recovery. Journal of Magnetic Resonance (1969) 92, 126 145 (1991).

59. Z. S. Saad et al., A new method for improving functional-to-structural MRI alignment using local Pearson correlation. Neuroimage 44, 839-848 (2009).

60. M. Weigel, Extended phase graphs: dephasing, RF pulses, and echoes-pure and simple. Journal of Magnetic Resonance Imaging 41, 266-295 (2015).

61. D. J. Wang et al., Multi-delay multi-parametric arterial spin-labeled perfusion MRI in acute ischemic stroke-comparison with dynamic susceptibility contrast enhanced perfusion imaging. Neurolmage: Clinical 3, 1-7 (2013).

62. D. C. Alsop et al., Recommended implementation of arterial spin-labeled perfusion MRI for clinical applications: a consensus of the ISMRM perfusion study group and the European consortium for ASL in dementia. Magnetic resonance in medicine 73, 102-116 (2015).

63. L. R. Huber et al., LayNii: A software suite for layer-fMRI. revision 1. BioRxiv (2020).

64. M. Waehnert et al., Anatomically motivated modeling of cortical laminae. Neuroimage 93, 210-220 (2014). 
65. B. Fischl, A. M. Dale, Measuring the thickness of the human cerebral cortex from magnetic resonance images. Proceedings of the National Academy of Sciences 97, 11050-11055 (2000).

66. J. B. Freeman, R. Dale, Assessing bimodality to detect the presence of a dual cognitive process. Behavior research methods 45, 83-97 (2013).

67. R. P. Larkin, An algorithm for assessing bimodality vs. unimodality in a univariate distribution. Behavior Research Methods \& Instrumentation 11, 467-468 (1979). 


\section{Figures}
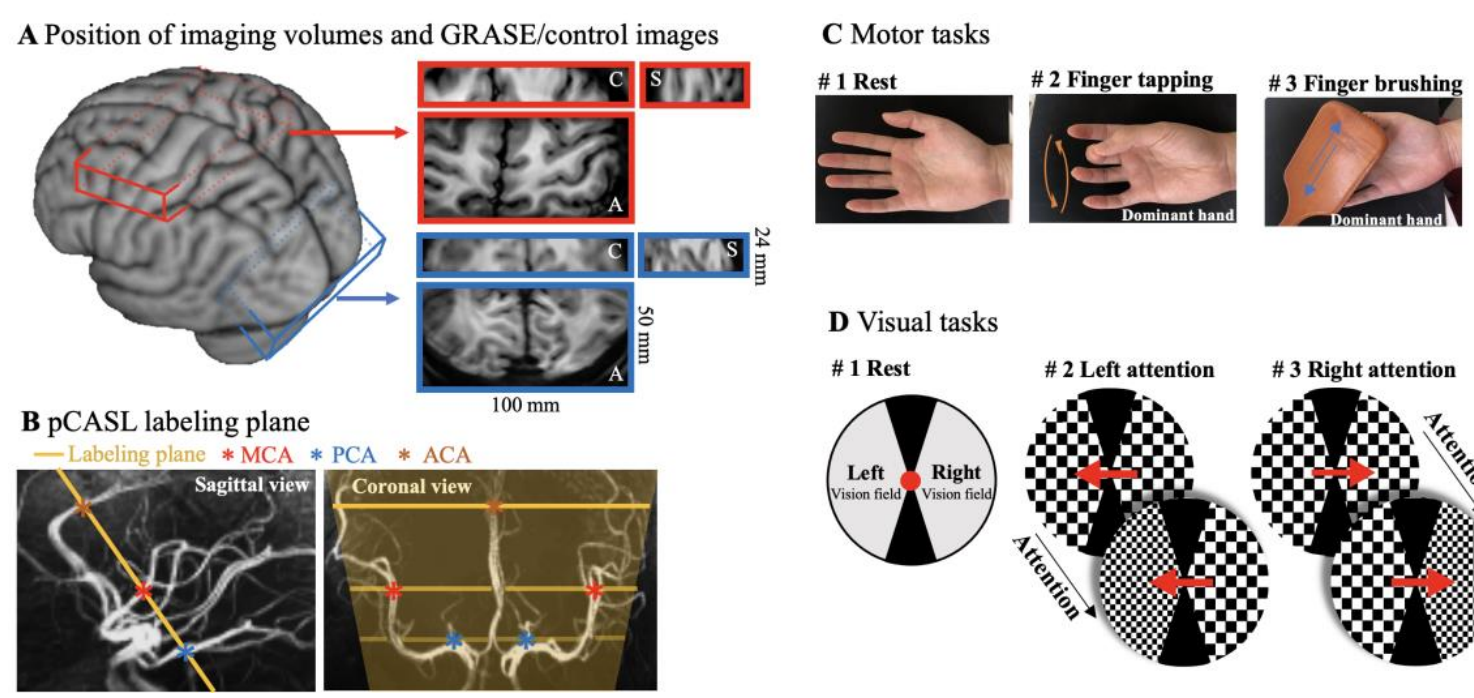

D Visual tasks

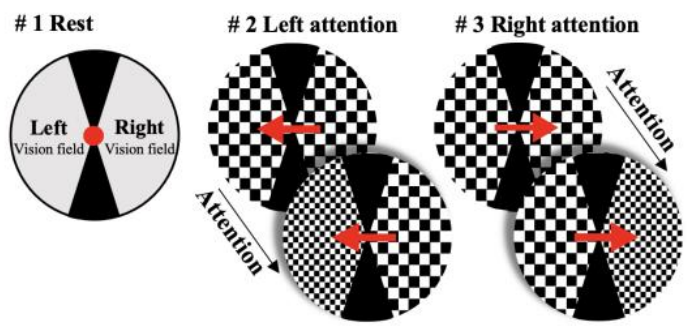

Figure 1. Demonstration of image acquisition and diagrams of functional tasks. A. Illustration of imaging volume on a 3D brain surface. A small FOV $\left(100 \times 50 \times 24 \mathrm{~mm}^{3}\right)$ covering the dominant motor cortex (red) or visual cortex (blue) was acquired with zoomed GRASE. Axial, coronal and sagittal views of GRASE images were shown in enlarged red and blue boxes, respectively. B. Illustration of pCASL labeling plane in sagittal (yellow line) and coronal (yellow lines and shade) views. Intracranial arteries were revealed by maximal-intensity-projection (MIP) of the T1w structural MRI and pCASL labeling plane was placed above the circle of Willis (CoW) and simultaneously perpendicular to the M3 segment of middle cerebral artery (MCA), P2 segment of posterior cerebral artery (PCA) and A2 segment of anterior cerebral artery (ACA). C. Diagram of motor tasks. Two motor tasks consisted unilateral sequential finger tapping and brushing of the dominant hand (frequency of $2 \mathrm{~Hz}$ ). D. Diagram of spatial visual attention tasks. A pair of counter-phase flickering ( $7.5 \mathrm{~Hz}, 50 \%$ contrast) square wave checkerboards patterns (2 cycles per degree) were presented to the left and the right side of fixation. During the stimulus presentation, the spatial frequency of the two checkerboard patterns changed 7 times randomly and independently. Subjects were asked to pay attention to the cued checkerboard to detect occasional spatial frequency change of the attended stimulus in left or right vision field in two visual task runs. 

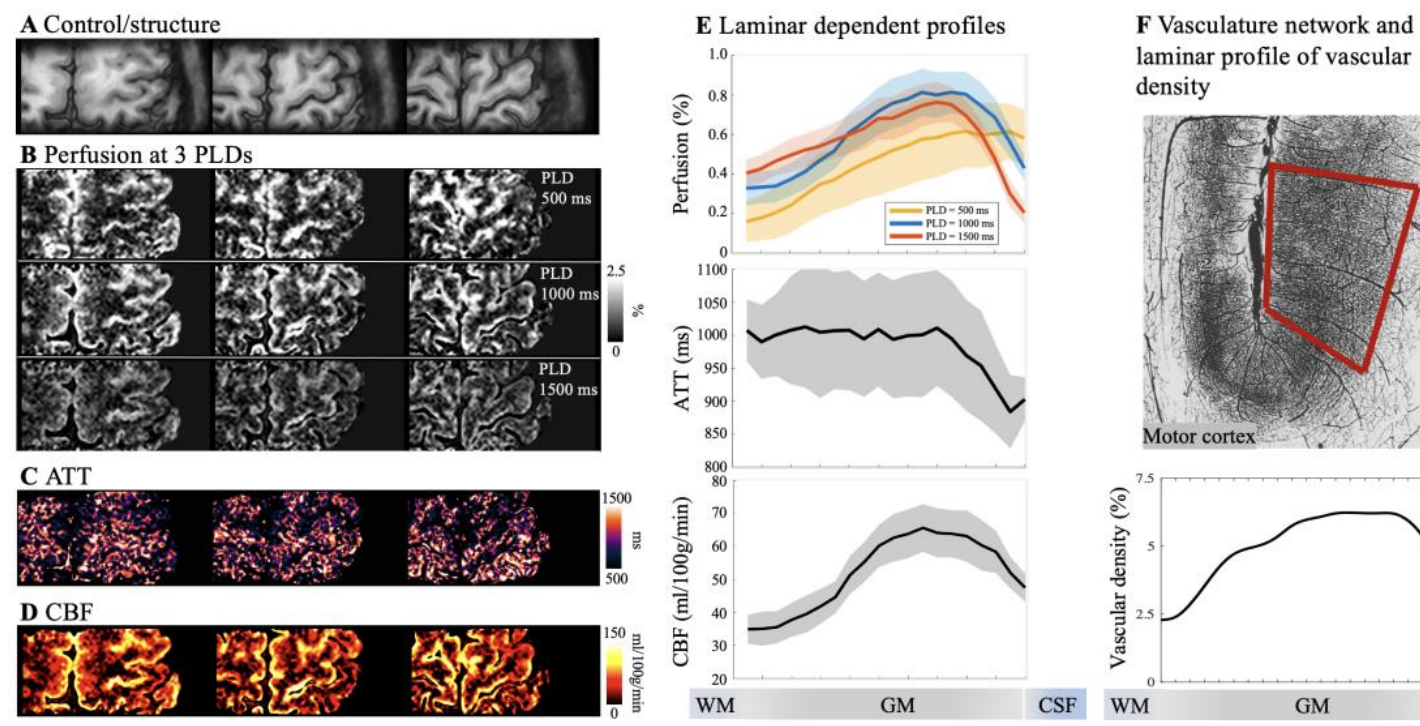
laminar profile of vascular

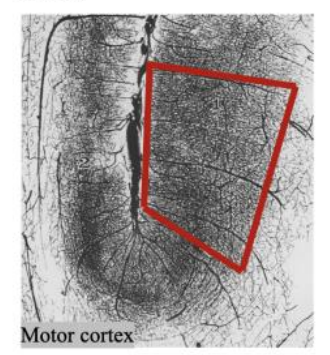

Figure 2. Resting state pCASL perfusion in motor cortex. A. Three slices of GRASE/control images demonstrating sufficient coverage for hand knob area in M1. B. Resting-state perfusion images acquired at three PLD of 500, 1000 and $1500 \mathrm{msec}$. Color bar indicates the percentage of perfusion signal in relation to $M_{0}$. Maps of ATT (C) and resting state CBF (D) were estimated from multi-PLD perfusion signals using a weighted-delay approach. E. Laminar profiles of multi-PLD perfusion signals, ATT and resting state CBF. Perfusion signal peaks at GM/CSF boundary at an early PLD of $500 \mathrm{msec}$, and the perfusion signal peak shifts towards middle layers at later PLDs of 1000 and $1500 \mathrm{msec}$. ATT was lower at GM/CSF boundary indicating fast transit to pial arteries and became homogeneous in middle and deep layers. CBF was higher in middle layers indicates higher microvascular density. Shaded areas indicate variations across subjects. F. Vasculature network obtained from specimen of huma brain in motor cortex (top row, frontal and parietal cortex on right and left side of central sulcus). Vessels are labeled in black and darker area indicate higher vascular density (Figure is adapted from (23)). Laminar profile of relative vascular density (bottom row) calculated from inverted image intensity within the red box. 

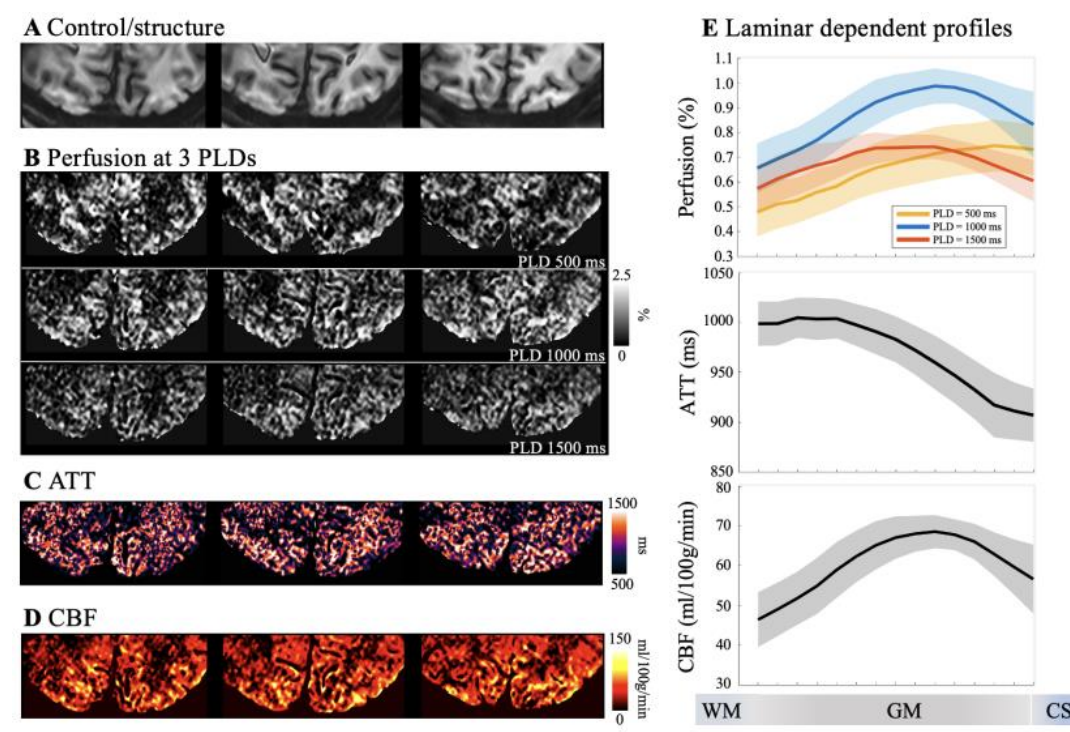
F Vasculature network and laminar profile of vascular density

Figure 3. Resting state pCASL perfusion in visual cortex. A. Three slices of GRASE/control images demonstrating sufficient coverage for V1. B. Resting-state perfusion images acquired at three PLD of 500,1000 and $1500 \mathrm{msec}$. Color bar indicates the percentage of perfusion signal in relation to Mo. Maps of ATT (C) and resting state CBF (D) were estimated from multi-PLD perfusion signals using a weighted-delay approach. E. Laminar profiles of multi-PLD perfusion signals, ATT and resting state CBF. Perfusion signal peaks at GM/CSF boundary at an early PLD of $500 \mathrm{msec}$, and the perfusion signal peak shifts towards middle layers at later PLDs of 1000 and $1500 \mathrm{msec}$. ATT was lower at GM/CSF boundary indicating fast transit to pial arteries and became homogeneous in middle and deep layers. CBF was higher in middle layers indicates higher microvascular density. Shaded areas indicate variations across subjects. F. Vasculature network obtained from specimen of huma brain in visual/striate cortex (top row). Vessels are labeled in black and darker area indicate higher vascular density (Figure is adapted from (23)). Striate cortex exhibit a distinct characteristic that most dense vascular network is found in the internal granular layer (layer 4). Laminar profile of relative vascular density calculated from inverted image intensity within the red box. 

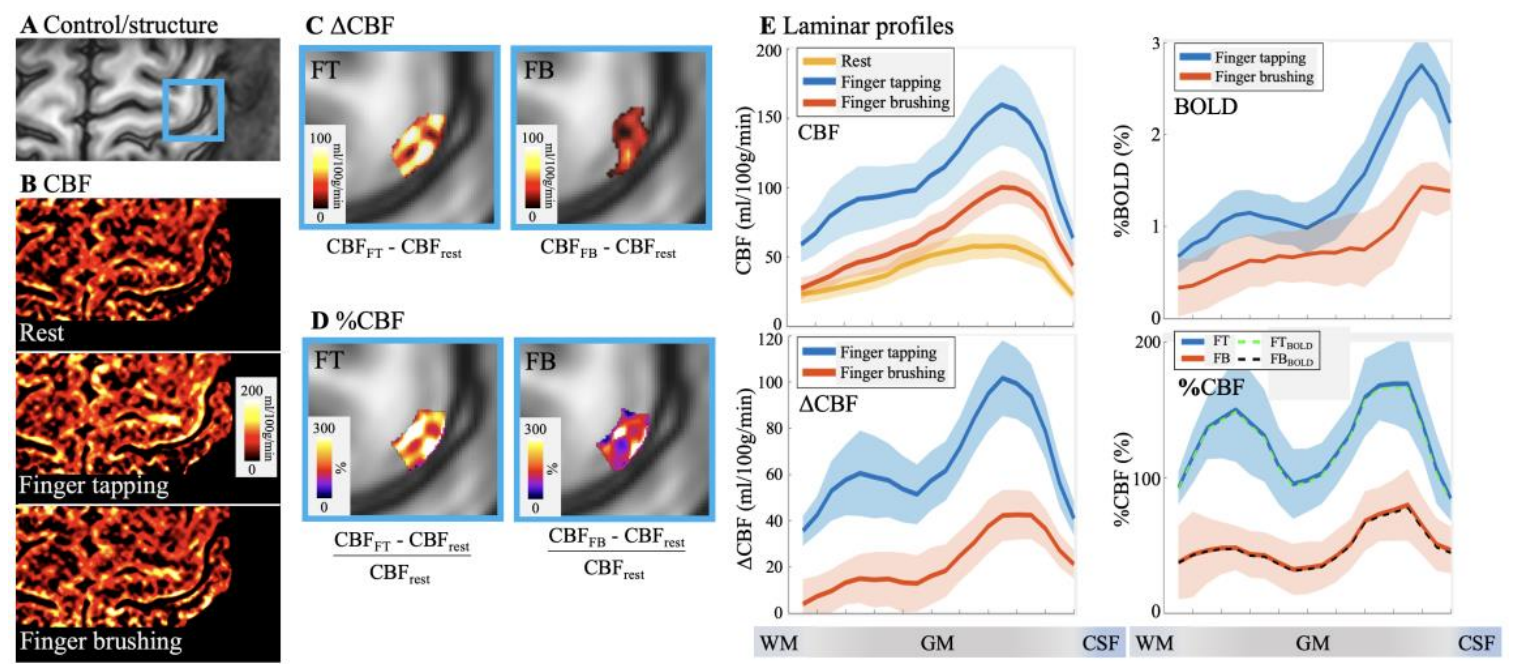

Figure 4. Motor tasks activated pCASL perfusion. A. GRASE image with hand knob area highlighted in blue box. B. CBF maps at rest (top), finger tapping and finger brushing. Absolute (C) and percentage (D) CBF increase evoked by the FT and FB tasks are highlighted in M1 hand knob area (blue boxes). E. Laminar profile of CBF at rest and two motor tasks (top left), percentage BOLD changes (top right), absolute (bottom left) and percentage (bottom right, dashed line (FT and $\mathrm{FB}_{\text {BOLD) }}$ was adjusted for BOLD effects) CBF changes induced by two motor tasks. Both FT and FB induced BOLD signal changes are mostly in superficial layers. FT induced BOLD response shows a weak increase in deep layers; however, the two-peak pattern was not significant $(P=0.47)$ due to the large BOLD signal change in superficial layers. FT induced CBF increase shows a 'twopeak' pattern $(P=0.011$ and $P<0.0002$ for significance test of two-peak pattern of $\triangle C B F$ and \%CBF, respectively) which corresponds to sensory input (superficial) and motor output (deep) layers respectively. FB induced CBF response shows a weaker increase in superficial layers which corresponds to exteroception sensory input and minimal motor output. Shaded areas indicate variations across subjects 


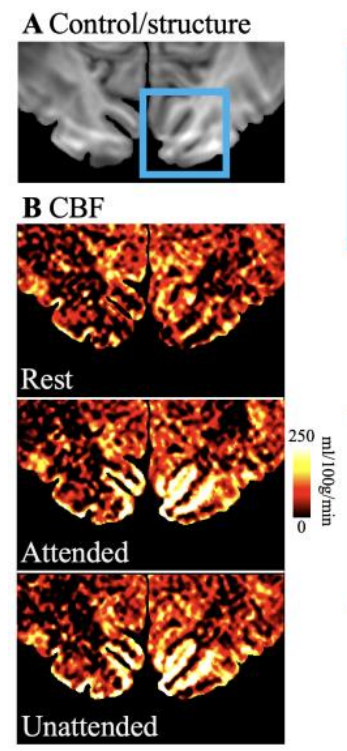

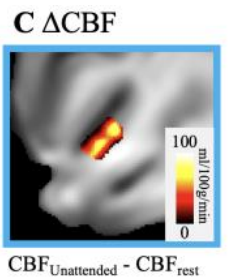

D $\% \mathrm{CBF}$

Unattended stimuli

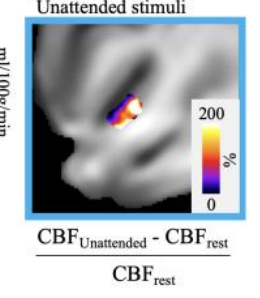

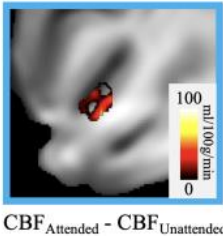

Attention modulation

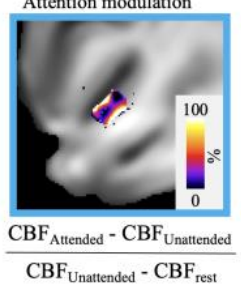

E Laminar dependent profiles
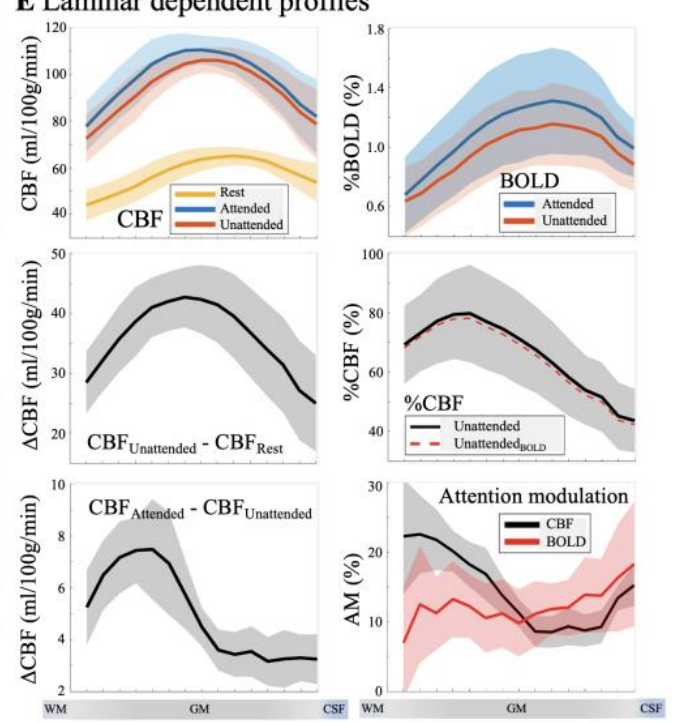

Figure 5. pCASL perfusion at spatial attention tasks. A. GRASE image with $\mathrm{V} 1$ highlighted in blue box. B. CBF maps at rest (top), attended and unattended visual stimuli. C. Absolute CBF increase evoked by unattended visual stimuli (left column) and spatial attention (right column) in V1. D. Relative CBF increase evoked by unattended visual stimuli (left column) and attention modulation (right column). E. Laminar profile of CBF at rest and with unattended and attended visual stimuli (top left), percentage BOLD changes (top right), absolute and relative CBF changes induced by unattended visual stimuli (middle panel, dashed lines were adjusted for BOLD effects), absolute CBF changes induced by spatial attention (bottom left) and CBF and BOLD response to attention modulation (bottom right). Both absolute and relative CBF increase evoked by unattended visual stimuli peak in middle layers. Absolute CBF increase evoked by spatial attention shows a large peak in deep layers while CBF profile of attention modulation shows a two-peak patten $(P=0.039$ of two-peak pattern significance test). BOLD profile of attention modulation is relatively homogeneous across layers with a slight increase towards $G M / C S F$ boundary ( $P=0.063$ of twopeak pattern significance test). Shaded areas indicate variations across subjects. 
bioRxiv preprint doi: https://doi.org/10.1101/2021.04.13.439689; this version posted April 14, 2021. The copyright holder for this preprint (which was not certified by peer review) is the author/funder, who has granted bioRxiv a license to display the preprint in perpetuity. It is made available under aCC-BY-NC-ND 4.0 International license. 